\title{
Adaptive Sliding Mode Control for A 2 DOF Magnetic Levitation System with Uncertain Parameters
}

\author{
Meng Duan \\ The Seventh Research Division and the Center for Information and Control, Beihang University (BUAA) \\ Beijing 100191, China \\ Yingmin Jia \\ The Seventh Research Division and the Center for Information and Control, Beihang University (BUAA) \\ Beijing 100191, China \\ E-mail: duanmeng90@126.com; ymjia@buaa.edu.cn \\ www.buaa.edu.cn
}

\begin{abstract}
This paper investigates the stability control for a 2 DOF magnetic levitation system with uncertain parameters and external disturbance. The electromagnetic forces between the magnets and coils are obtained by numerical calculation. Then, an adaptive sliding mode controller is proposed to deal with the parameter uncertainties in the system matrices. The robust stability of the closed-loop system is proved by Lyapunov stability theory. Simulation results are presented to verify the effectiveness of the proposed control strategy.
\end{abstract}

Keywords: adaptive sliding mode control, magnetic levitation, numerical calculation, uncertain input

\section{Introduction}

In recent years, magnetic levitation technology becomes more and more important in the modern industry, like high precision transportation, because magnetic levitation technology can eliminate the friction, vibrations and wear caused by contact. And these machines have to move in multiple degrees of freed-om(DOF). In Ref. 1-2, two kinds of planar motors using Halbach permanent magnet arrays which can move in a large horizontal plane have been proposed. Ref. 3 design-ed a long stroke 6 DOFs direct drive machine, the mover was constructed by symmetric linear magnet arrays and control currents generated by manufactured PCBs.

However, large rotation ranges are limited by the distribution of magnetic field in the above mentioned technologies, forces and torques in six DOFs can not be calculated sufficiently accurately in real time. ${ }^{4}$ To this end, Ref. 5 proposed a novel single-deck planar maglev stage, its electromagnetic forces and torques are analyzed by the ANSOFT software. Refs. 6 also used numerical calculation method to obtain accurate electromagnetic forces and torques, and large rotation ranges have been realized.

Inspired by those works, this paper mainly focus on the problem of stability control for a 2-DOF magnetic levitation system subjects to parameter uncertainties. The contributions include that

(i) In the modeling process of electromagnetic forces, the errors between the real position and preset one are described as the uncertainties of control matrix.

(ii) Based on the sliding mode method, an adaptive controller is designed to guarantee the robust 
stability of the closed-loop system which has uncertain parameters and external disturbances.

This paper is organized in six sections. Section 2 describes electromagnetic forces numerical calculation model; Section 3 analyses the uncertain parameters of system dynamic model; Section 4 presents the controller design. Simulation results are presented in section 5 . And the conclusions are given in section 6.

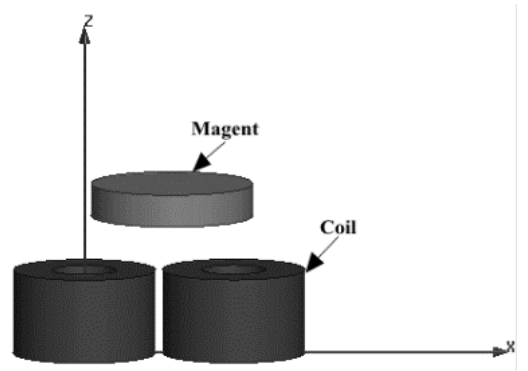

Fig. 1. Magnetic levitation system

\section{Electromagnetic Force Model}

In this paper, we investigate a 2 DOFs magnetic levitation system that consists of one disk magnet and two cylindrical coils shown in Fig.1, Assume that the levitated magnet can only move through $\mathrm{x}$-axis and $\mathrm{z}$ axis but can not rotate.

Generally, electromagnetic forces are calculated by Lorentz's formula at a fixed relative position between the magnet and coils. The total forces are calculated as the volume integrals:

$$
F=\iiint J \times B_{m} d V=\frac{I}{A} \iiint a \times B_{m} d V
$$

where $J$ is the vector of the current density of coils, $I$ is the coil current, $A$ is the cross-sectional area of the coil, $a$ is the direction of the current, $B_{m}$ is the vector of magnetic field density generated by the magnet and it is the function of the relative position of the magnet and the coils, $V$ is the volume of coils. From Eq. (1) we can get that the electromagnetic forces are proportional to the magnitude of coil currents at a fixed relative position. So we can write Eq. (1) as follow:

$$
F=\hat{b} I
$$

where $\hat{b}=\frac{I}{A} \iiint a \times B_{m} d V$.
In practice, the impact between coils can be ignored, so the forces generated by each coil are independent, the total forces can be calculated simply by adding the forces generated by each coil. The forces can be written as:

$$
F=\sum \hat{b}_{i} I_{i}=\hat{B} I \quad i=1,2
$$

where $I=\left[I_{1}, I_{2}\right]^{T}, \hat{B}=\left[\hat{b}_{1}, \hat{b}_{2}\right]^{T}$.

Because the forces generated by each coil current are proportional and independent, a numerical model can be established that only includes one magnet and one coil, the electromagnetic forces are calculated in this model on each preset position along $\mathrm{x}$-axis and $\mathrm{z}$ axis with a unit ampere of current to get $\hat{b}_{i}$, then use Eq. (3) to get $F$.

But the preset positions in numerical calculation model are discrete, when the magnet moves on the positions that have not been numerical calculated, we can not find the matrix $\hat{B}$. In Ref. 6, B. Peter uses nearest-neighbor positions to replace real positions, so the $\hat{B}$ is not certain at some positions, in this paper, the uncertainties of $\hat{B}$ are introduced as $\Delta \hat{B}$, obviously, it is bounded, so the electromagnetic forces can be written as:

$$
F=\left(\hat{B}_{o}+\Delta B\right) I
$$

where $\hat{B}_{o}$ is the nominal part of $\hat{B}$.

\section{Model Analysis}

The dynamic model of the magnetic levitation system can be derived by using Newton's Law as follow:

$$
\begin{aligned}
& F_{x}=\sum f_{x i}=m \ddot{x} \\
& F_{z}=\sum f_{z i}=m \ddot{z}+m g \quad i=1,2
\end{aligned}
$$

where $m$ is the mass of magnet, $g$ is acceleration of gravity. Choose $X=\left[x_{1}, x_{2}, x_{3}, x_{4}\right]=[x, \dot{x}, z, \dot{z}]$, apply Eq. (4) to Eq. (5), the dynamic model can be expressed as follow:

$$
\dot{X}=f(X)+(B+\Delta B) I
$$

where $f(X)=\left[x_{2}, 0, x_{4}, g\right]^{T}, I=\left[I_{1}, I_{2}\right]^{T}, \Delta B=\Delta \hat{B} / m$, $B=\left[\begin{array}{cccc}0 & \hat{b}_{11} & 0 & \hat{b}_{21} \\ 0 & \hat{b}_{12} & 0 & \hat{b}_{22}\end{array}\right]^{T} / m=\left[\begin{array}{cccc}0 & b_{11} & 0 & b_{21} \\ 0 & b_{12} & 0 & b_{22}\end{array}\right]^{T}$. 
Simultaneously consider the uncertain parameters of system dynamic model and external disturbances, write dynamical model as follow:

$$
\dot{X}=f_{o}(X)+\Delta f(X)+(B+\Delta B) I+w
$$

where $f_{o}(X)$ is the nominal part of $f(X), \Delta f(X)$ is the uncertain part of $f(X), w$ is external disturbance, assume that both of them are bounded.

\section{Adaptive Sliding Mode Controller Design}

Define the desired state as $X_{d}=\left[x_{d 1}, x_{d 2}, x_{d 3}, x_{d 4}\right]^{T}$, the tracking error can be written as:

$$
E=\left[e_{1}^{T}, e_{2}^{T}\right]^{T}=X_{d}-X
$$

where $e_{1}=[E(1), E(2)]^{T}, e_{2}=[E(3), E(4)]^{T}$.

Because the levitated magnet moves along two axes, the sliding-surface function can be introduced as:

$$
s=\left[s_{1}, s_{2}\right]^{T}=\left[c_{1}^{T} e_{1}, c_{2}^{T} e_{2}\right]^{T}
$$

where $c_{1}=\left[c_{11}, c_{12}\right]^{T}, c_{2}=\left[c_{21}, c_{22}\right]^{T}$. The constant $c_{1}$ and $c_{2}$ are chosen to be positive to make sure that the polynomial $c_{11} \lambda+c_{12}$ and $c_{11} \lambda+c_{12}$ is Hurwitz.

Differentiating $s$ yields:

$$
\dot{s}=c^{T} f(X)-c^{T} \dot{X}_{d}+c^{T} B_{o} I+W(X, I)
$$

where $c=\left[\begin{array}{ll}c_{1} & \\ & c_{2}\end{array}\right], W(X, I)$ is the lumped uncertain parameters and external disturbance:

$$
W(X, I)=\left[W_{1}, W_{2}\right]^{T}=c^{T} \Delta f(X)+c^{T} \Delta B I+c^{T} w
$$

in practice, the input coil currents are bounded, we can guarantee that $W(X, I)$ is bounded.

We design the control input $I$ to be:

$$
I=I_{o}+I_{s}
$$

where

$$
\begin{aligned}
& I_{o}=-\left(c^{T} B_{o}\right)^{-1}\left(c^{T} f(X)-c^{T} \dot{X}_{d}\right) \\
& I_{s}=-\left(c^{T} B_{o}\right)^{-1} \hat{P} \operatorname{sgn}(s)=-\left(c^{T} B_{o}\right)^{-1}\left[\begin{array}{l}
\hat{P}_{1} \operatorname{sgn}\left(s_{1}\right) \\
\hat{P}_{2} \operatorname{sgn}\left(s_{2}\right)
\end{array}\right]
\end{aligned}
$$

and $\hat{P}_{1}$ and $\hat{P}_{2}$ are adjustable parameters, and the adaptive law is:

$$
\dot{\hat{P}}=\left[\frac{1}{\rho_{1}}\left\|s_{1}\right\|, \frac{1}{\rho_{2}}\left\|s_{2}\right\|\right]^{T}
$$

where $r_{1}$ and $r_{2}$ are the adaptation gain and both are positive. Assume that $P_{d 1}$ and $P_{d 2}$ are the terminal solution of $\hat{P}_{1}$ and $\hat{P}_{2}$ which satisf $\left|W_{1}\right| \leq P_{d 1},\left|W_{2}\right| \leq P_{d 2}$, respectively.

Choose the adaption error as:

$$
\tilde{P}=\left[\begin{array}{l}
\hat{P}_{1}-P_{d 1} \\
\hat{P}_{2}-P_{d 2}
\end{array}\right]=\left[\begin{array}{c}
\tilde{P}_{1} \\
\tilde{P}_{2}
\end{array}\right] .
$$

Define a Lyapunov candidate function as:

$$
V=\frac{1}{2} s^{T} s+\frac{1}{2} \tilde{P}^{T}\left[\begin{array}{cc}
\rho_{1} & 0 \\
0 & \rho_{2}
\end{array}\right] \tilde{P} .
$$

The time derivative of the Lyapunov candidate function can be found to be:

$$
\begin{aligned}
\dot{V} & =s^{T} \dot{s}+\tilde{P}^{T}\left[\begin{array}{cc}
\rho_{1} & 0 \\
0 & \rho_{2}
\end{array}\right] \dot{\tilde{P}} \\
& =s^{T}\left(c^{T} f(X)-c^{T} \dot{X}_{d}+c^{T} B_{o} I+W(X, I)\right)+\left[\begin{array}{c}
\rho_{1}\left(\hat{P}_{1}-P_{d 1}\right) \\
\rho_{2}\left(\hat{P}_{2}-P_{d 2}\right)
\end{array}\right]^{T} \dot{\tilde{P}} \\
& =s^{T}\left(W(X, I)-\left[\begin{array}{l}
\hat{P}_{1} \operatorname{sgn}\left(s_{1}\right) \\
\hat{P}_{2} \operatorname{sgn}\left(s_{2}\right)
\end{array}\right]+\left[\begin{array}{l}
\hat{P}_{1}-P_{d 1} \\
\hat{P}_{2}-P_{d 2}
\end{array}\right]^{T}\left[\begin{array}{l}
s_{1} \operatorname{sgn}\left(s_{1}\right) \\
s_{2} \operatorname{sgn}\left(s_{2}\right)
\end{array}\right]\right. \\
& =s_{1} W_{1}+s_{2} W_{2}-\left|s_{1}\right| P_{d 1}-\left|s_{2}\right| P_{d 2} \leq 0 .
\end{aligned}
$$

Because $V \in L_{2}, \dot{V}$ is bounded, according to Barbalat Lemma, $V$ would converge to zero, so the tracking error $E$ would converge to zero. In order to alleviate the input chattering caused by the $\operatorname{sgn}\left(s_{i}\right)$ in Eq. (14), the saturation function $\operatorname{sat}\left(s_{i} / \phi\right)$ is used to replace $\operatorname{sgn}\left(s_{i}\right),{ }^{7}$ where $\phi$ is constant.

\section{Simulation Results}

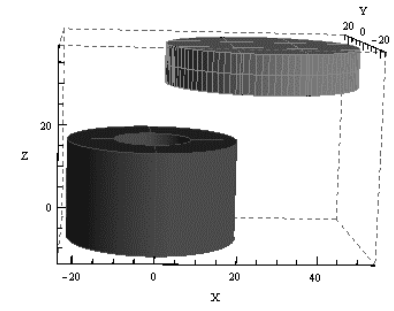

Fig. 2. Numerical calculation model. 

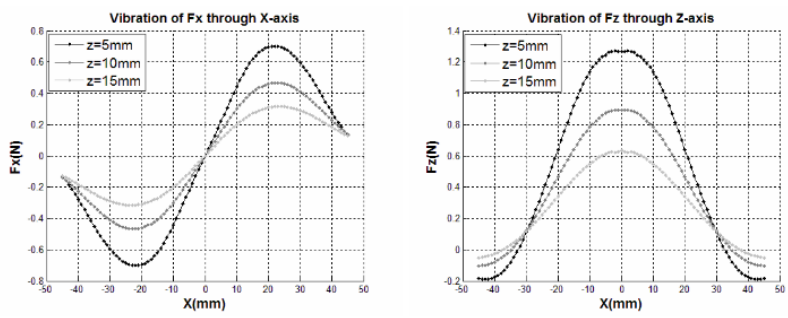

Fig. 3. Vibration of electromagnetic forces along $\mathrm{x}$-axis with different air gaps
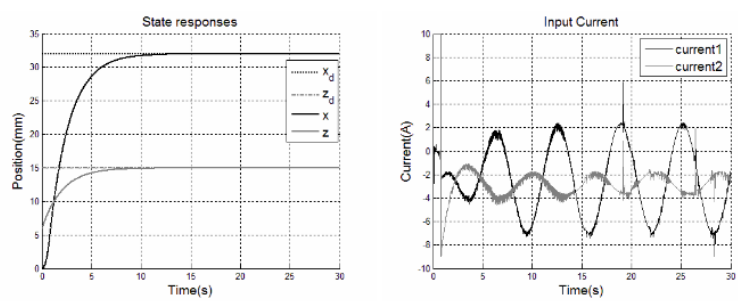

Fig. 4. Position tracking response performance.
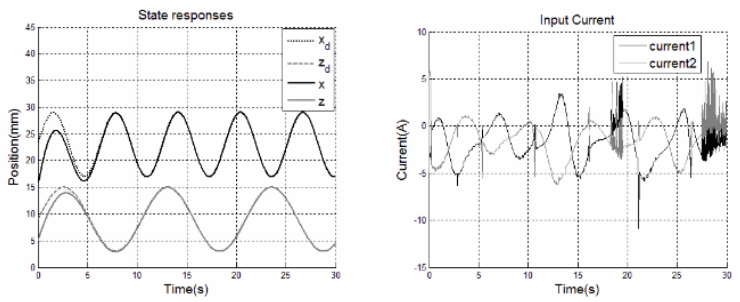

Fig. 5. Trajectory tracking response performance

In this paper, the electromagnetic force model is calculated by Radia which is a free 3D magnetostatics computation software package developed by ESRF and running in Mathematica. The numerical calculation model established in Radia is shown in Fig. 2. The coil in the numerical calculation model is $26 \mathrm{~mm}$ in height, $20 \mathrm{~mm}$ in the inside diameter and $44 \mathrm{~mm}$ in the outside diameter. The current density in the coil is $0.97 \mathrm{~A} / \mathrm{mm}^{2}$. The magnet is cylindrical with $50 \mathrm{~mm}$ in diameter and $10 \mathrm{~mm}$ in height, its material is $\mathrm{NdFeB}$ and the remnant magnetization is $1.22 T$. The calculation sampling inter$\mathrm{val}$ is $1 \mathrm{~mm}$ along $\mathrm{z}$-axis and $\mathrm{x}$-axis. Figure. 3 shows that vibration of electromagnetic forces when the magnet moves along $\mathrm{x}$-axis with different air gaps.

In this section, two typical simulation results which including the trajectory and position tracking are presented. In both two simulations, $\Delta f(X)$ is supposed to be $\left[0.3 \sin t\left(\left|x_{1}\right|+\left|x_{2}\right|\right), 0,0.2 \cos t\left(\left|x_{1}\right|+\left|x_{2}\right|\right), 0\right]^{T}, w$ is designed as $[0, \sin t, 0, \sin t]^{T}$, and the parameters of the controller are designed as: $c_{1}=c_{2}=[0.5,1], \rho_{1}=\rho_{2}=0.5$, $\phi=0.1, \hat{P}_{1}(0)=30, \hat{P}_{2}(0)=70$.

The position tracking response performance of the 2-DOF magnetic levitation system is shown in Fig. 4, where the levitated magnet can track the desire position $x=32 \mathrm{~mm}$ and $z=15 \mathrm{~mm}$. From the tracking curve shown in Fig. 4, we can see that the magnet can reach and keep balance at the desired position with small currents.

The trajectory tracking response performance is shown in Fig. 5, from the simulation results, we can see that the levitated magnet can track the desired trajectories $x=6 \sin t+22 \mathrm{~mm}$ and $z=6 \sin (0.6 t)+9 \mathrm{~mm}$.

\section{Conclusions}

In this paper, electromagnetic forces obtained by the method of numerical calculation, which brings the uncertainties to the control matrix, to deal with this, an adaptive sliding model controller has been developed and applied to the 2-D maglev system, the simulations confirmed the efficacy of the proposed controllers.

\section{Acknowledgements}

This work was supported by the National Basic Research Program of China (973 Program: 2012CB821200, 2012CB821201) and the NSFC (61134005, 61327807, 61521091, 61520106010).

\section{References}

1. H.-S. Cho and H.-K. Jung, Analysis and design of synchronous permanent-magnet planar motors, IEEE T ENERGY CONVER. 17(4)(2002)492 -499.

2. J. W. Jansen, Magnetically levitated planar actuator with moving magnets, IEEE T IND APPL. 46(5)(2008)11081115.

3. X. Lu and I.-U.-R. Usman, 6D direct-drive technology for planar motion stages, CIRP ANN-MANUF TECHN. 61(1)(2012)359-362.

4. M. B. Khamesee and E. Shameli, Regulation technique for a large gap magnetic field for 3D non-contact manipulation, Mechatronics. 15(2005)1073-1087.

5. Y.-C. Lai, Y.-L. Lee and J.-Y. Yen, Design and servo control of a single-deck planar maglev stage, IEEE $T$ $M A G N$. 43(6)(2007)2600 -2602. 
6. B. Peter and D. Michael, Magnetic levitation over large translation and rotation ranges in all directions, IEEEASME T MECH. 18(1)(2013) 44 -52.

7. Y.-J. Huang, T. C. Kuo and S.-H. Chang, Adaptive sliding-mode control for nonlinear systems with uncertain parameters, IEEE T SYST MAN CY B. $38(2)(2008) 534-539$. 